\title{
Collaboration During Visual Search
}

by

\author{
Kelly A. Malcolmson \\ A thesis \\ presented to the University of Waterloo \\ in fulfillment of the \\ thesis requirement for the degree of \\ Master of Arts \\ in \\ Psychology
}

Waterloo, Ontario, Canada, 2006

CKelly Malcolmson 2006 


\section{Author's Dedaration for E lectronic Submission of a Thesis}

I hereby declare that I am the sole author of this thesis. This is a true copy of the thesis, including any required final revisions, as accepted by my examiners.

I understand that my thesis may be made electronically available to the public. 


\begin{abstract}
Three experiments examine how collaboration influences visual search performance. Working with a partner or on their own, participants reported whether a target was present or absent in briefly presented search displays. The search performance of individuals working together (collaborative pairs) was compared to the pooled responses of the individuals working alone (nominal pairs). Collaborative pairs were less likely than nominal pairs to correctly detect a target and they were less likely to make false alarms. Signal detection analyses revealed that collaborative pairs were more sensitive to the presence of the target and had a more conservative response bias than the nominal pairs. This pattern was observed when the search difficulty was increased and when the presence of another individual was matched across pairs. The results are discussed in the context of task sharing, social loafing and current theories of visual search.
\end{abstract}




\section{Acknowledgements}

I would like to give special thanks to my supervisor Daniel Smilek for his guidance, enthusiasm, flexibility, and constant encouragement.

Thanks to Mike Reynolds, our collaborator on this project, for his help with generating ideas and solutions, for his programming expertise, and for assistance with writing.

I would also like to thank all the members of the Smilek lab for making the lab such a fun place to be! In particular thanks to Jonathan Carriere for always making my life easier by providing simple and brilliant solutions to the many problems I encounter, and to Donna Kwan for keeping me sane by listening to my endless stories.

Also a special thanks to Heather Wilk, Molly Pottruff and Julie Lounsbury for their assistance with data collection. 


\section{Table of Contents}

Author $\rrbracket$ s Declaration for Electronic Submission of a Thesis ......................................................................ii

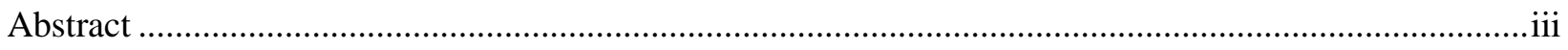

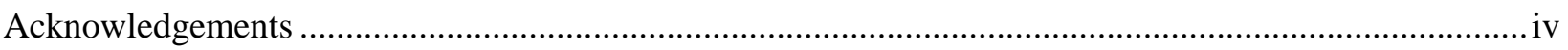

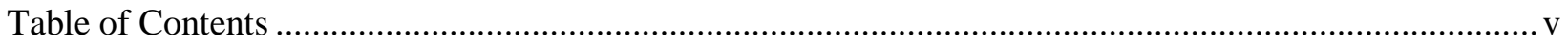

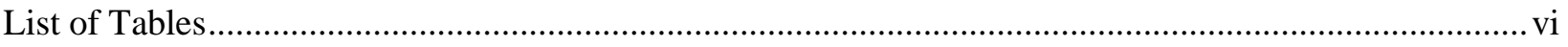

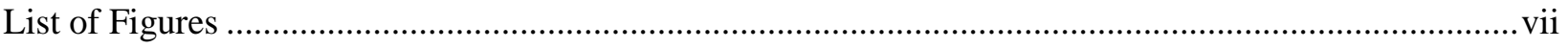

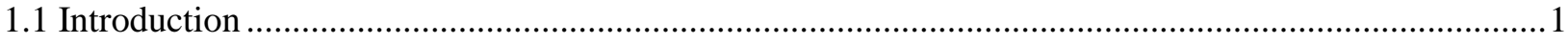

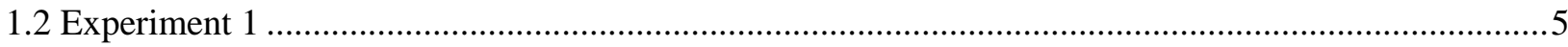

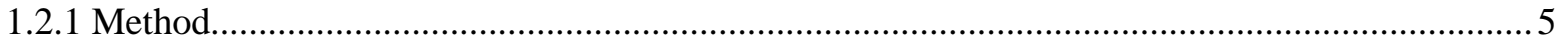

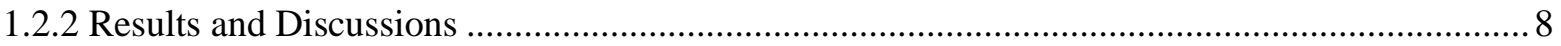

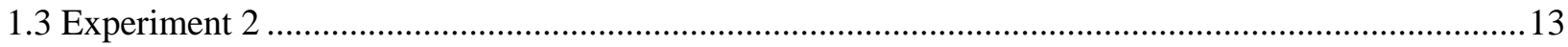

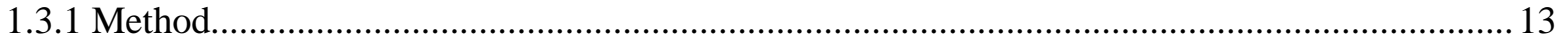

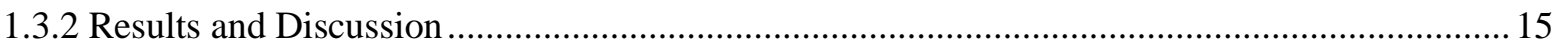

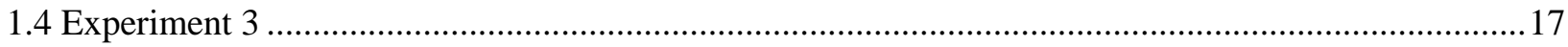

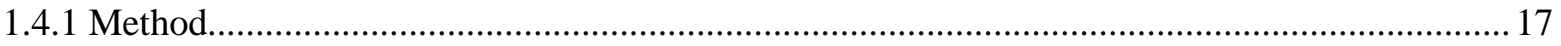

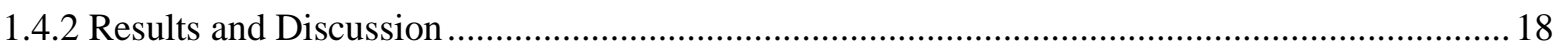

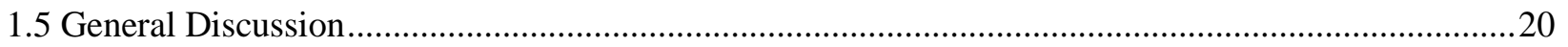

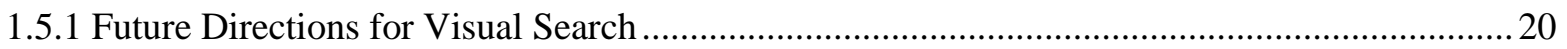

1.5.2 Future Directions for Collaborative Search...................................................................... 23

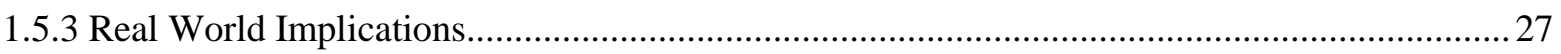

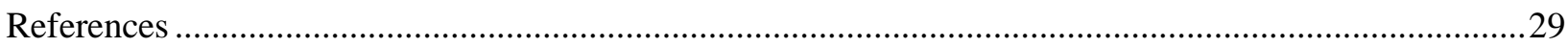

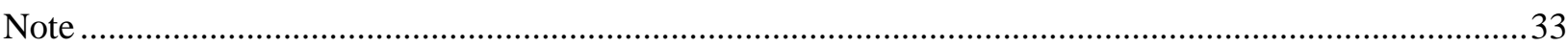

Appendix A : Mean Proportion of Hits, Proportion of False Alarms, Sensitivity and Response Bias in the Collaborative and Nominal Conditions

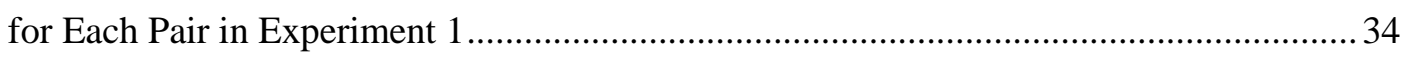

Appendix B : Mean Proportion of Hits, Proportion of False Alarms, Sensitivity and Response Bias in the Collaborative and Nominal Conditions

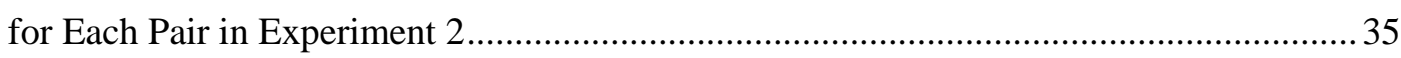

Appendix C : Mean Proportion of Hits, Proportion of False Alarms, Sensitivity and Response Bias in the Collaborative and Nominal Conditions

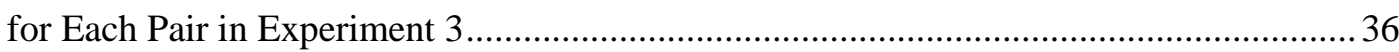




\section{L ist of T ables}

Table 1. Mean proportion hits, proportion false alarms, sensitivity ( $\left.\mathrm{A}^{\prime}\right)$ and response

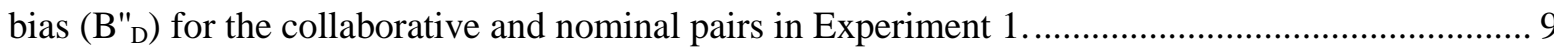

Table 2. Mean proportion hits, proportion false alarms, sensitivity (A') and response

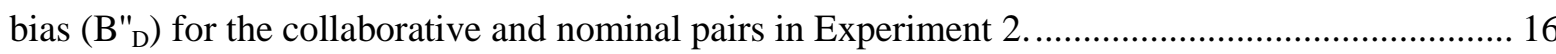

Table 3. Mean proportion hits, proportion false alarms, sensitivity $\left(\mathrm{A}^{\prime}\right)$ and response bias $\left(\mathrm{B}_{\mathrm{D}}\right)$ for the collaborative and nominal pairs in Experiment 3. 


\section{List of Figures}

Figure 1. Examples of (A) target present and (B) target absent displays used in

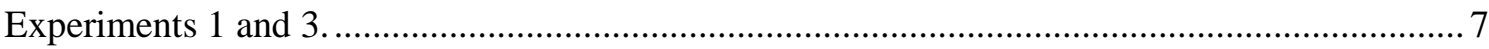

Figure 2. Sensitivity (A') for detecting the target in the collaborative and nominal

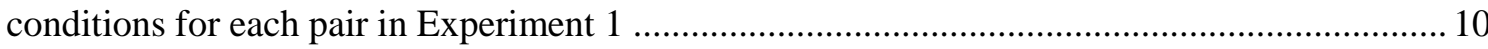

Figure 3. Examples of (A) target present and (B) target absent displays used in

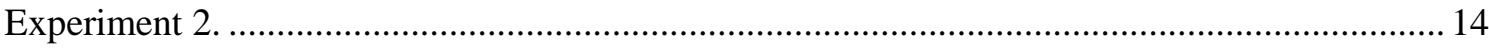




\subsection{Introduction}

Visual search is often a collaborative process. For instance, consider how couples might search for a street address while driving. This task is often distributed among the individuals in the automobile, with one person searching on the left side of the road while the other person searches on the right. Or, consider medical diagnosis. Experts could collaboratively search X-rays to arrive at a decision about the presence of a tumor. Finally, consider security in public places such as airports. Before boarding an airplane, bags can be searched by multiple individuals and X-ray images of luggage are sometimes scanned by two observers working together to detect weapons and illegal substances. In the present work I examine how the search performance of two individuals working together compares to the pooled search performance of two individuals working alone.

Though collaborative search is common in everyday life, studies of visual search have primarily focused on how individuals search in isolation from others. This focus is understandable given that the general goal has been to isolate the specific cognitive (e.g., Duncan \& Humphreys, 1989; Treisman \& Gelade, 1980; Wolfe, 1994), computational (e.g., Bundesen, 1990; Humphreys \& Muller, 1993), behavioral (e.g., Findlay \& Gilchrist, 1998; Hooge \& Erkelens, 1996; Zelinsky \& Sheinberg, 1997) and brain (e.g., Luck \& Hillyard, 1994; Rolls \& Deco, 2002) mechanisms that underlie visual search. These mechanisms presumably reside within an individual and so it makes sense to study individuals in isolation. The result of this focus, however, has been the general neglect of the impact of social collaboration on search performance.

The importance of studying how collaboration impacts search performance is also reinforced by a growing body of evidence from studies of navigation (e.g., Hutchins, 1995), collaborative memory (e.g., Weldon \& Bellinger, 1997; Ross, Spencer, Linardatos, Lam \& Perunovic, 2004), joint attention (e.g., Tomasello, 1995), and joint action (e.g., Sebanz, Bekkering, \& Knoblich, 2006), which show that social collaboration and interaction can have a substantial impact on basic cognitive and brain processes. These 
studies reflect a more general trend in which cognition is seen as being part of a more complex system that includes the whole physical body and the local physical and social environment (see Clark, 1999; Kingstone et al., 2003; Thelen \& Smith, 1994). The main assumption of this “embodied” or "situated cognition” approach is that a complete understanding of various aspects of cognition (such as attention) will include an understanding of the interaction between individuals and their physical and social environments.

Following this general line of inquiry three studies are reported investigating how social collaboration influences visual search. Pairs of individuals performed a simple visual search task both together and on their own. Samples of the search displays used in the experiments are shown in Figure 1. Participants searched for a "backwards C" which had a solid line on the right side, among distractor items, which had gaps on both sides. The target was present in half of the displays and participants were required to press a key on the keyboard if a target was present, and to withhold making a response when the target was absent. The visual search displays were presented briefly (e.g., $400 \mathrm{~ms}$ ) and participants had a maximum of $2000 \mathrm{~ms}$ to respond (see Klein \& Farrell, 1989). In this way, it was possible to measure correct target detection (hits) and false target detection (false alarms) and to calculate estimates of target sensitivity and response bias.

There were two critical conditions: collaborative and nominal. In the collaborative condition, participants sat side-by-side in front of a computer screen, shared a single "target present" response button and performed the visual search task together. Collaborative pairs were encouraged to discuss how they might best perform the task together. In the nominal condition, each participant independently performed the search task on a separate computer. Within a given nominal pair, each member was presented with exactly the same sequence of displays so that it was possible to combine the responses from the two individuals on a trial-per-trail basis. In both collaborative and nominal conditions, a response was designated as "present” when one or both participants indicated the target was present and a response was 
designated as "absent” when both participants indicated the target was absent. In this way, the pooled performances of the nominal pairs were directly comparable to the performance of the collaborative pairs (see Ross et al., 2004). Specifically, hits, false alarms, target sensitivity and response bias across the collaborative and nominal pairs are compared.

How might collaboration influence search performance? One possibility is that collaboration will lead to task sharing (e.g., dividing up the search display) and therefore better search performance for collaborative pairs than for nominal pairs. More specifically, based on a shared understanding of the task derived through dialog between the members of the pair (i.e., script sharing; see Jack \& Roepstroff, 2002; Roepstroff \& Frith, 2004), each individual could execute top-down control to selectively process nonredundant aspects of the display (e.g., one person looks on the right side of the screen while the other searches the left). Compared to performance of a nominal pair, such collaboration should result in more accurate target detection (i.e., more hits) and more withheld responses to target absent displays (i.e., less false alarms), resulting in a greater sensitivity to the targets. This is because nominal pairs would likely have considerably more redundancy in the way in which they processed each display relative to collaborative pairs. There is also no reason to believe that, according to this view, there should be any difference in response bias across groups. Of course task sharing would depend heavily on the extent to which people have top-down control over visual search processes. Such top-down control would be very difficult if, for instance, visual search has no memory (Horowitz \& Wolfe, 1998) or if top-down control is very limited (see Wolfe, 1994).

Alternatively, there is good reason to believe that collaboration may hurt search performance. There is an overwhelming consensus across a wide range of research areas including memory (e.g., Basden, Basden, Bryner, Thomas, 1997; Weldon \& Bellinger, 1997), brainstorming (e.g., Harkins \& Petty, 1982; Harkins \& Jackson, 1985), vigilance (e.g., Harkins \& Petty, 1982; Harkins \& Szymanski, 1989) and joint action (e.g., rope pulling; Ingham, Levinger, Graves, \& Peckham, 1974), that the 
performance of groups is often poorer than the pooled performance of individuals working alone. This counterintuitive phenomenon is broadly referred to as social loafing (see Karau \& Willimas, 1993 for a review). Given the prevalence of this outcome, it certainly seems plausible that nominal pairs might outperform collaborative pairs during search. Poorer collaborative performance could be reflected in fewer hits, more false alarms and a lower sensitivity for collaborative than nominal pairs; social loafing does not predict how collaborative and nominal pairs might differ in terms of response bias.

A final possibility is that collaboration will not influence sensitivity to targets but will only influence response bias. Support for this possibility comes from a recent study of how collaboration influences recognition performance (Ross et al., 2004). In this study spouses generated shopping lists and then completed a recognition test either together or separately. The recognition test included items from the shopping list as well as novel items. The nominal pair was formed by pooling the independent judgments of the two individuals such that if one or both members recognized an item, it was designated as recognized. If neither of the individuals recognized the item, it was designated as not recognized. The results showed no difference across collaborative and nominal pairs in the sensitivity of memory as measured by d' (i.e., no collaborative inhibition). However, collaborative pairs made fewer false alarms than did the nominal pairs indicating that collaborative pairs are less likely to designate an item as being recognized (i.e., have a more conservative response bias). If collaboration in visual search follows the same principles as it does in recognition tasks, then it will not influence sensitivity to targets but rather lead to a more conservative response bias.

These alternatives are first evaluated in a relatively easy search task in which the target is easily distinguishable from the distractors (Experiment 1). The generality of the results of Experiment 1 are then evaluated in a more difficult search context (Experiment 2). Finally, the last experiment (Experiment 3) rules out the possibility that any observed differences between collaborative and nominal pairs were due to the mere presence of another individual in the collaborative condition. 


\subsection{Experiment 1}

\subsubsection{Method}

Participants. Forty-eight undergraduates (24 pairs) from the University of Waterloo participated in a one-hour session in exchange for one course credit. All participants had normal or corrected-tonormal vision.

Stimulus Displays. Examples of the types of stimulus displays used in the experiment are shown in Figure 1. One half of the displays contained the target object (target present), while the other half did not (target absent). The target (a "backwards C”) and distractor (an "upside-down V” on top of a "rightside up V”) differed only in terms of the features on the right side of each object; the target always had a solid line connecting the bottom and top pieces, while there was a gap between these pieces on the distractors. The set size was varied randomly from trial to trial within participant such that there were

either 8 or 16 items in each display ${ }^{1}$. The items in each display were presented in random locations of an imaginary $5 \times 5$ grid. The search objects measured $1 \mathrm{~cm}\left(1^{\circ}\right)$ in width and $1.4 \mathrm{~cm}\left(1.4^{\circ}\right)$ in height at a viewing distance of $57 \mathrm{~cm}$. The gap between the top and bottom pieces was $0.5 \mathrm{~cm}\left(0.5^{\circ}\right)$. Each search display grid measured $17 \mathrm{~cm}\left(17^{\circ}\right)$ in width and $15 \mathrm{~cm}\left(15^{\circ}\right)$ in height. The stimulus displays were presented by E-Prime (Psychology Software Tools, www.pstnet.com) on ViewSonic PT775 monitors that were driven by Pentium based computers.

Procedure. Each trial of the experiment began with a fixation cross (+) presented for $1500 \mathrm{~ms}$. The offset of the fixation cross coincided with the onset of a search display which was presented for 300, 400 or $500 \mathrm{~ms}$. The exposure durations $(300,400$, or $500 \mathrm{~ms}$ ) were varied across participants (i.e. 8 pairs at each exposure duration). The offset of the search display was followed by a blank display which remained on screen for a maximum of $2000 \mathrm{~ms}$ or until the participant responded. Participants were required to press the spacebar on the keyboard if the target was present and to withhold a response if the target was absent. Responses were accepted during both the stimulus display screen and the blank 
display. By presenting the displays only briefly it was possible to measure both correct target detection (hits) as well as false target detection (false alarms) and to derive measures of sensitivity and response bias. After a response was given or the maximum time elapsed, a feedback display was presented for $1500 \mathrm{~ms}$ indicating whether the response was correct or incorrect. All together there were 16 practice trials and 120 experimental trials in each of the collaborative and nominal conditions.

Participants completed the task once as part of a collaborative pair and once as part of a nominal pair. When in the collaborative condition, participants were instructed to complete the search task as a team and to try to think of a strategy that would take advantage of the fact that there were two of them searching the displays. The collaborative pairs were seated in front of a single computer screen and shared a response key (the spacebar). A response was coded as "present” if one or both members of the pair pressed the response key. A response was coded as "absent” if both members withheld a response. Every 40 trials, participants were instructed to: “Take some time to evaluate how well your search strategy is working. Discuss with your partner whether you want to change the strategy you are using”. This was done to encourage collaboration. In the nominal condition, each person performed the visual search task independently on separate computers. Each pair received exactly the same displays in the same order. Response coding was done on a trial-by-trial basis; a response was counted as "present” if on a given trial one or both individuals pressed the response key and it was coded as "absent” if both members withheld a response. To match for the breaks given in the collaborative condition for discussion of strategy, similar breaks were given in the nominal condition. Every 40 trials, each participant was instructed to: "Take some time to evaluate how well your search strategy is working. Decide whether you want to change the strategy you are using”.

The order in which the pairs participated in the collaborative and nominal conditions was counterbalanced across pairs. Half of the pairs performed the task as a collaborative pair first, followed by the nominal condition in which they each performed the task on their own. The other half of the pairs 
A)

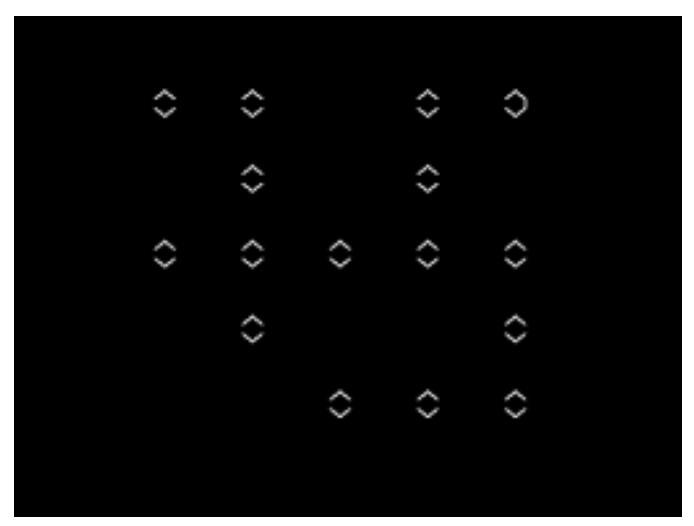

B)

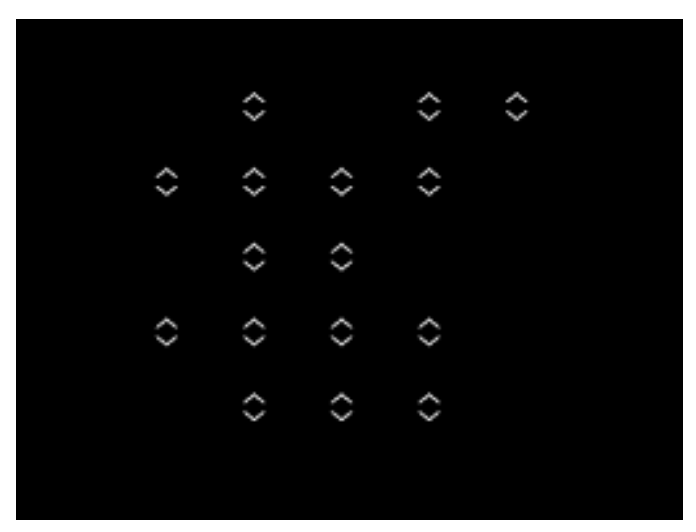

Figure 1. Examples of (A) target present and (B) target absent displays used in Experiments 1 and 3. 
first completed the nominal condition followed by the collaborative condition. To ensure that participants did not receive the same set of displays in the collaborative and nominal conditions, two different sets of displays were created. The display sets were counterbalanced across the collaborative and nominal search conditions.

At the end of both the collaborative and nominal conditions, subjective reports were obtained. Each individual was asked by the experimenter about the strategy they used and how they searched the display screen. The experimenter took notes on the participants $\square$ verbal reports.

\subsubsection{Results and Discussions}

Table 1 shows the overall search performance of the collaborative and nominal pairs in terms of hits, false alarms, sensitivity $\left(\mathrm{A}^{\prime}\right)$ and response bias $\left(\mathrm{B}_{\mathrm{D}}{ }_{\mathrm{D}}\right)$. Nonparametric estimates of sensitivity and response bias (see Donaldson, 1992) were used because there was only one measure of hits and false alarms in each of the conditions precluding the calculation of parametric estimates. Each of the measures (hits, false alarms, sensitivity and response bias) was submitted to a repeated measures t-test assessing the effects of collaboration (collaborative pairs vs. nominal pairs).

As can been seen in the table, collaborative pairs had fewer hits than did nominal pairs, $\mathrm{t}(23)=$ $6.39, p<0.001$. However, collaborative pairs also had a much lower proportion of false alarms than the nominal pairs, $\mathrm{t}(23)=9.70, \mathrm{p}<0.001$. Taking into account both the hits and false alarms, collaborative pairs showed a greater sensitivity $\left(\mathrm{A}^{\prime}\right)$ for detecting the target than did the nominal pairs, $\mathrm{t}(23)=4.47, \mathrm{p}<$ 0.001. This pattern was highly consistent across pairs (see Figure 2). In addition, the collaborative pairs

had a more conservative response bias $\left(\mathrm{B}{ }_{\mathrm{D}}\right)$ than did the nominal pairs, $\mathrm{t}(23)=10.23, \mathrm{p}<0.001$, meaning that collaborative pairs were less likely to respond that the target was present. The mean proportion of hits, proportion of false alarms, sensitivity and response bias for each pair in the collaborative and nominal conditions in Experiment 1 are shown in Appendix 1. 
Table 1. Mean proportion hits, proportion false alarms, sensitivity $\left(\mathrm{A}^{\prime}\right)$ and response bias $\left(\mathrm{B}_{\mathrm{D}}\right)$ for the collaborative and nominal pairs in Experiment 1. The difference scores represent collaborative performance minus nominal performance.

\begin{tabular}{lcccc}
\hline Condition & Hits & False Alarms & Sensitivity & $\begin{array}{c}\text { Response Bias } \\
\text { (A') }\end{array}$ \\
\hline Collaborative & .806 & .161 & .888 & .168 \\
Nominal & .936 & .499 & .818 & -.856 \\
\hline Difference & -.129 & -.338 & .070 & 1.024 \\
& $(\mathrm{p}<.001)$ & $(\mathrm{p}<.001)$ & $(\mathrm{p}<.001)$ & $(\mathrm{p}<.001)$ \\
\hline
\end{tabular}




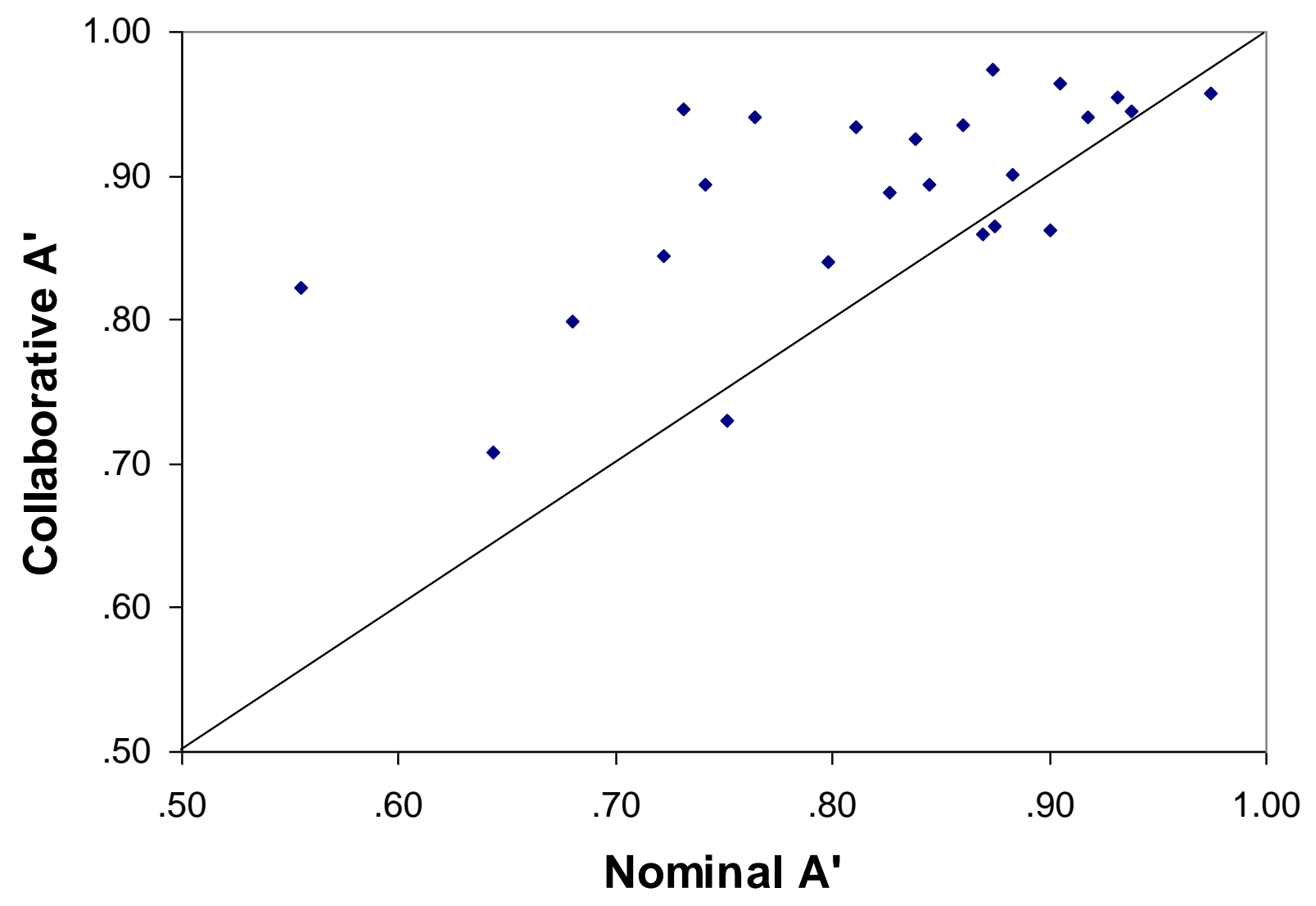

Figure 2. Sensitivity (A') for detecting the target in the collaborative and nominal conditions for each pair in Experiment 1. The diagonal line indicates equivalence between conditions. 
The results of Experiment 1 are particularly interesting because they show a pattern that is much different than those predicted by the accounts discussed at the outset. The results are only partially consistent with task sharing. Consistent with the notion that collaborative pairs effectively divided the task, collaborative pairs were found to have fewer false alarms and showed a greater sensitivity to the targets. Inconsistent with the straightforward prediction of this view, however, the collaborative group also had fewer hits. A clear weakness of the naive task sharing view is that it failed to make strong predictions about how collaboration might influence response bias. Social loafing also failed to predict the whole pattern of results. Though the lower hit rate in collaborative pairs is consistent with social loafing, the lower false alarm rate and the greater sensitivity in collaborative pairs is not consistent with this view. And again, social loafing failed to make strong predictions about how collaboration might influence response bias. Finally, the recent recognition memory literature also failed to predict the entire pattern of results. Consistent with the recognition literature, collaborative pairs made fewer false alarms and had a more conservative response bias. However, inconsistent with this literature, the collaborative pairs also had a greater sensitivity than did nominal pairs.

Subjective reports of individuals were consistent with the idea that the collaborative advantage is, at least in part, due to task sharing between members of the pair. Of the 24 collaborative pairs, 23 chose to divide up the search space. The most common solution was splitting the screen down the centre; 19 of the 24 pairs $(79.2 \%)$ split the screen so that one person searched on the left half while the other person searched the right half. Two pairs (8.3\%) split screen horizontally with one person searching the top half and the other person focusing on the bottom half. Of the remaining three pairs, two chose to split the screen in some combination of the two former strategies, while the one pair that did not divide up the task chose to have each member of the pair randomly search the entire screen. The finding that pairs reported parsing each search display so as to reduce redundant searching and the observation that the majority chose to split the screen vertically rather than horizontally, have important implications for theories of 
visual search. Namely, the findings highlight the need for a more nuanced specification of the sorts of “top-down” strategies that are implemented during search. 


\subsection{Experiment 2}

The purpose of Experiment 2 was to determine whether the pattern of results obtained in Experiment 1 would generalize to a more difficult search task. To make the search task more difficult, the visual similarity between the target and distractor stimuli was increased (Duncan \& Humphreys, 1989). Examples of the search displays used in Experiment 2 are shown in Figure 3. As can be seen in the figure, the target was the same as in Experiment 1. The only difference in the displays was the shape of the distractors; in Experiment 2 the gap size on the right hand side of the distractors was reduced to increase target-distractor similarity relative to Experiment 1 . To ensure that participants were still able to complete the task, exposure durations of the search displays were increased. As in Experiment 1 performance between pairs working together (collaborative condition) with their pooled performance when working apart (nominal condition) was compared.

\subsubsection{M ethod}

Participants. Forty-eight undergraduates (24 pairs) from the University of Waterloo participated in a one-hour session for one course credit. All participants had normal or corrected-to-normal vision.

Stimulus Displays. Examples of the stimulus displays are shown in Figure 3. Displays were created in the same manner as Experiment 1. The only difference was that in the present displays the distractor stimuli were more visually similar to the target. Instead of having the full gap on the right side, the distractors only had a gap size of $0.2 \mathrm{~cm}\left(0.2^{\circ}\right)$.

Procedure. The procedure was the same as in Experiment 1, with the exception that the exposure duration of the search displays was increased $(600,800$, and $1000 \mathrm{~ms})$ to compensate for the increase in search difficulty incurred by the increase in target-distractor similarity. 
A)

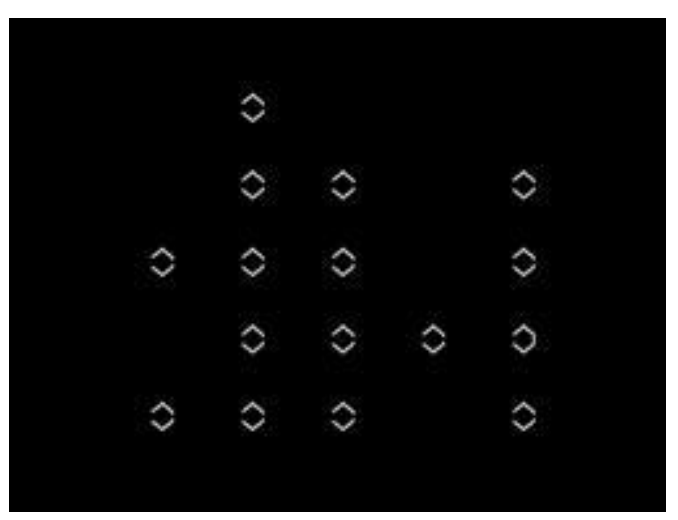

B)

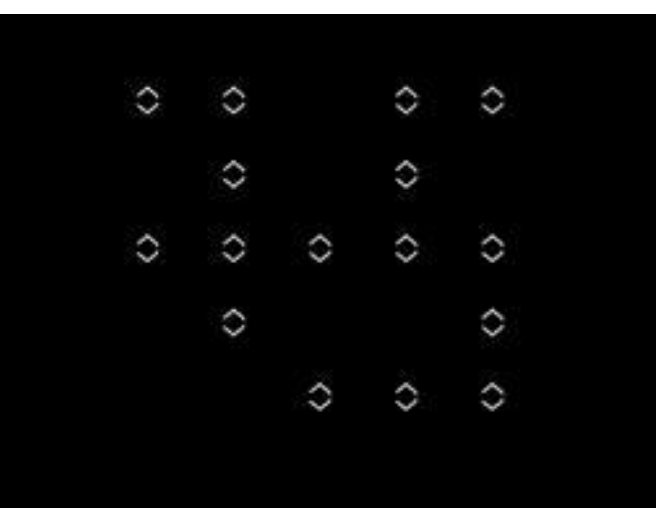

Figure 3. Examples of (A) target present and (B) target absent displays used in Experiment 2. 


\subsubsection{Results and Discussion}

Table 2 shows the overall search performance for the nominal and collaborative pairs in terms of hits, false alarms, sensitivity $\left(\mathrm{A}^{\prime}\right)$ and response bias $\left(\mathrm{B}_{\mathrm{D}}{ }_{\mathrm{D}}\right)$. When each of the measures (hits, false alarms, sensitivity and response bias) was submitted to a repeated measures t-test assessing the effects of collaboration (collaborative pairs vs. nominal pairs), a similar pattern of results to Experiment 1 was obtained. The collaborative pairs again had fewer hits, $\mathrm{t}(23)=-8.43, \mathrm{p}<.001$, and fewer false alarms, $t(23)=-5.57, p<.001$, than the nominal pairs. Again when taking into account both the hits and false alarms, collaborative pairs showed a greater sensitivity (A') for detecting the target than did the nominal pairs, $t(23)=3.28, p<.005$. A similar response bias effect can be seen in which the collaborative pairs are more conservative; the collaborative pairs were again less likely to respond that the target was present, $t(23)=10.32, p<.001$. The mean proportion of hits, proportion of false alarms, sensitivity and response bias for each pair in the collaborative and nominal conditions in Experiment 2 are shown in Appendix 2.

Thus, the overall pattern of results from Experiment 1 was replicated. The findings of a collaborative advantage for sensitivity and a more conservative response bias in the collaborative condition appear to generalize to a more difficult search. 
Table 2. Mean proportion hits, proportion false alarms, sensitivity $\left(\mathrm{A}^{\prime}\right)$ and response bias $\left(\mathrm{B}_{\mathrm{D}}{ }_{\mathrm{D}}\right)$ for the collaborative and nominal pairs in Experiment 2. The difference scores represent collaborative performance minus nominal performance.

\begin{tabular}{lcccc}
\hline Condition & Hits & False Alarms & Sensitivity & \multicolumn{2}{c}{ Response Bias } \\
\hline Collaborative & .672 & .143 & .844 & (B' ${ }^{\prime}$ ) \\
\hline Nominal & .818 & .473 & .758 & -.503 \\
\hline Difference & -.146 & -.330 & .086 & 1.017 \\
& $(\mathrm{p}<.001)$ & $(\mathrm{p}<.001)$ & $(\mathrm{p}<.005)$ & $(\mathrm{p}<.001)$ \\
\hline
\end{tabular}




\subsection{Experiment 3}

In Experiment 3 I sought to examine whether the differences in search between the collaborative pairs and nominal pairs in Experiments 1 and 2 were due to social presence. There are good reasons to believe that the mere presence of another individual could influence performance and lead to the differences between collaborative and nominal pairs (e.g., Zajonc, 1965). For instance, the mere presence of another individual could have made people more vigilant, leading to greater sensitivity when searching with a partner in a collaborative pair than when searching alone as part of a nominal pair. The mere present of a partner could also have led to a more conservative response bias in collaborative pairs. Indeed, participants commented that when they searched with a partner and responded "present” on target absent trials, they "felt bad" and would apologize to their partners.

To evaluate the effects of social presence during search, pairs again completed the search task used in Experiment 1 either separately or together. This time however, when participants completed the task separately, an experimenter sat beside each participant. Participants were informed that the experimenter would be monitoring their performance. In this way social presence was matched for across collaborative and nominal conditions. If the differences in search performance between the collaborative and nominal pairs found in Experiments 1 and 2 were simply due to the mere presence of another individual in the collaborative condition, then the differences should now be eliminated.

\subsubsection{M ethod}

Participants. Forty-eight undergraduates (24 pairs) from the University of Waterloo participated in a one-hour session for one course credit. All participants had normal or corrected-to-normal vision. Stimulus Displays. The displays from Experiment 1 (Figure 1) were used in this experiment. Procedure. The procedure was identical to Experiment 1 with the exception that an experimenter sat beside each individual while they performed the task separately in the nominal condition. 


\subsubsection{Results and Discussion}

Having matched for social presence across the collaborative and nominal pairs a very similar pattern of results to those found in Experiments 1 and 2 was obtained. Overall search performance in terms of hits, false alarms, sensitivity $\left(\mathrm{A}^{\prime}\right)$ and response bias $\left(\mathrm{B}^{\prime \prime}\right)$ can be seen in Table 3. As in the two previous experiments, the collaborative pairs had fewer hits, $\mathrm{t}(23)=4.70, \mathrm{p}<0.001$, and false alarms, $\mathrm{t}(23)=7.79, \mathrm{p}<0.001$, than the nominal pairs. In addition, the collaborative pairs were again more sensitive at detecting the target, $\mathrm{t}(23)=3.68, \mathrm{p}<0.001$, than the nominal pairs. The collaborative pairs were again more conservative than the nominal pairs, $\mathrm{t}(23)=7.25, \mathrm{p}<0.001$. In these respects, the results of Experiment 3 replicate the findings of Experiments 1 and 2 and suggest that the search differences between collaborative and nominal pairs found in Experiments 1 and 2 cannot simply be attributed to differences in social presence across pairs. The mean proportion of hits, proportion of false alarms, sensitivity and response bias for each pair in the collaborative and nominal conditions in Experiment 3 are shown in Appendix 3. 
Table 3. Mean proportion hits, proportion false alarms, sensitivity $\left(\mathrm{A}^{\prime}\right)$ and response bias $\left(\mathrm{B}_{\mathrm{D}}{ }_{\mathrm{D}}\right)$ for the collaborative and nominal pairs in Experiment 3. The difference scores represent collaborative performance minus nominal performance.

\begin{tabular}{lcccc}
\hline Condition & Hits & False Alarms & Sensitivity & $\begin{array}{c}\text { Response Bias } \\
\text { (A') }\end{array}$ \\
\hline Collaborative & .806 & .178 & .884 & .036 \\
Nominal/Social & .913 & .432 & .833 \\
\hline Difference & -.107 & -.254 & .051 & .749 \\
& $(\mathrm{p}<.001)$ & $(\mathrm{p}<.001)$ & $(\mathrm{p}<.002)$ & $(\mathrm{p}<.001)$ \\
\hline
\end{tabular}




\subsection{General Discussion}

Three experiments investigated how collaboration affects visual search performance. In all experiments, the performance of pairs of individuals collaborating on the search task (collaborative pair) was compared to the pooled performance of two individuals completing the search task separately (nominal pair). The results of Experiments 1 and 2 showed that the collaborative pairs had fewer hits and fewer false alarms than the nominal pairs. When both the hits and false alarms were taken into account, the collaborative pairs were more sensitive to the presence of the target and they had a more conservative response bias than did nominal pairs. These findings were different than what was expected based on task sharing in collaborative pairs and based on studies of social loafing (see Karau \& Williams, 1993 for a review) and collaborative recognition memory (e.g., Ross et al., 2004).

Experiment 3 ruled out the possibility that the pattern of results described above were simply due to the mere presence of another individual in the collaborative condition. Social facilitation theory (Zajonc, 1967) predicts that the mere presence of others facilitates an individual $\square$ s performance on simple tasks. However in the present work, when social presence was matched across the collaborative and nominal conditions, the same pattern of results were obtained, suggesting that the differences obtained between the two conditions cannot be attributed solely to social facilitation.

\subsubsection{F uture Directions for V isual Search}

The present findings have important implications for theories of visual search and provide a new way to study top-down processes in visual search. Most current theories of visual search maintain that search efficiency is determined by both bottom-up and top-down processes (Duncan \& Humphreys, 1989; Treisman \& Gelade, 1980; Wolfe, 1994). However, the experimental focus has been largely on bottomup processes, and in particular, how various features and physical aspects of display items influence search efficiency (see Treisman \& Gelade, 1980; Wolfe, 1994; Wolfe \& Horowitz, 2004; but see Wolfe, 
Friedman-Hill, Stewart \& O\Connell, 1992). In addition, when top-down processes are implemented in models of visual search, they rarely include the sorts of complex strategies that people seem to use in everyday search settings.

For instance, consider the Guided Search Model reported by Wolfe (1994). Wolfe (1994) notes that control of the deployment of attention can be exogenous, based on the properties of the visual stimuli, or endogenous, based on the demands of the "user" of the visual system. However the model focuses primarily on implementing the exogenous properties of the visual stimuli guiding attention in a bottom-up fashion to specific locations of the visual field. The model describes an early, pre-attentive stage of the visual system, in which all locations of the visual field are processed in parallel. During this stage, only a limited amount of information about basic visual features can be extracted. The visual stimulus is first filtered through “categorical channels”, such as color and orientation. The output from these filters produces feature maps, which contain the information required to identify the specific colors, orientations, depths and so forth that are present in the visual input. This information is then used to restrict the deployment of subsequent limited-capacity stages, in which more complex tasks are performed over a smaller portion of the visual field.

According to the model, top-down processes help guide attention when the featural properties of search target are not sufficiently distinctive from the distractors to support guidance by bottom-up processes. A good example of this sort of top-down guidance is Wolfe, Friedman-Hill, Stewart and O\Connell[ $\llbracket$ (1992) study of top-down guidance for a target defined by orientation. Wolfe and colleagues (1992) found that participants would categorize stimuli into a small number of categories according to their orientation (e.g. steep, shallow, tilted left, tilted right). Therefore instead of searching for $22^{\circ}$ or $56^{\circ}$ targets among $0^{\circ}$ or $90^{\circ}$ distractor items, which might tax the visual system, the stimuli can be categorized so that the task becomes a simple search for a tilted target among untilted distractors. In the Guided Search Model, top-down activation is accomplished by selecting the category for each feature (e.g., "red" 
for color and "shallow" for orientation in a search for red horizontal lines) that best differentiates the target from the distractors. This is accomplished by weighting the categories according to the uniqueness of each target category, and the difference between the categories of the target and the distractors. This sort of categorization is one top-down process suggested by the Guided Search Model to make search more efficient.

For present purposes, the important point raised by Wolfe】s model is that the top-down processes implemented in the model are rather primitive. Indeed, it does not even seem that these processes are under conscious control. This is in stark contrast to the top-down strategies described in the present work, which clearly operate at a very deliberate and conscious level.

The present study of collaborative search highlights the importance of investigating top-down processing that operates at a very deliberate, flexible and conscious level. The finding that collaborative pairs had greater sensitivity to targets than nominal pairs and the observation that collaborative pairs reported dividing the search display between the two members of the pair, suggests that effective collaboration involves coordination of top-down strategies such that pairs are able to selectively process non-redundant aspects of the displays. In fact, collaborative search may be an important tool for (a) identifying the sorts of top-down strategies people use in various contexts and (b) directly testing whether these reported strategies are effective.

Further along these lines, the present findings are consistent with Roepstroff and Frith $\llbracket$ s (2004; Jack \& Roepstroff, 2002) suggestion that “top-down processes” can be understood as the "sharing of scripts” among individuals. Though many models of visual search include top-down processes, it remains unclear where these top-down processes originate. Top-down control is often represented in idealized models as a controlling homunculus located in a mythical brain region with outputs coming out of it, but no inputs. Roepstroff and colleagues suggest that in many studies, “the origin of the „executive top凸” is not inside the brain of the participant but rather "in the mind of the experimenter" (Roepstroff \& Frith, 
2004, p. 192). In other words, the top-down plans emerge out of the shared representation of the task that is communicated through dialogue between the experimenter and the participant (see also Pickering \& Garrod, 2004). Most studies of visual search do not discuss the dialogue between experimenter and participant because it is seen as a necessary precondition for conducting the experiment and not as a part

of the experiment proper. In contrast, the present studies of collaborative search highlight the importance of shared dialogue because collaborative dialog is included in the experiment proper. The two members of the collaborative pair discuss a strategy and it is through this dialogue that a common, shared representation of the task emerges. Each individual will then implement this script while performing the search task. This aspect of collaborative search opens a new avenue for future studies of visual search.

In future studies I plan to monitor dialogue between collaborative pairs to investigate what sorts of other task divisions are possible and useful in various types of search displays. In the present study, the majority of the collaborative pairs chose to divide the displays spatially into a left and right side with fewer dividing the display from top to bottom. I will use different kinds of displays such as ones with multiple targets or multiple types of distractors, as well as complex-real world stimuli, and analyze what types of top-down strategies people use when searching. For example, in a multiple target paradigm, will pairs choose to have one member search for one type of target, while the other one searches for the other type? Or will pairs continue to choose to divide the search display spatially, as they did in the present search task? Based on the types of strategies pairs choose to implement, I will be able to see what types of control is possible and which strategies take priority. This will provide us with a better understanding of top-down conscious control in visual search, which will in turn lead to new theories of visual search.

\subsubsection{F uture Directions for Collaborative Search}

Having established a new method for measuring sensitivity to targets and response bias during collaborative search, future research should also focus on how these measures are affected by specific changes in search context. Knowing how sensitivity and response bias during collaborative search vary 
across situations is a necessary step towards forming a theory of collaborative search. Based on the available research regarding how people work in groups (e.g., Karau \& Williams, 1993; Williams, Harkins, \& Latane, 1981; Ingham et al., 1974; Collaros \& Anderson, 1969; Andersson \& Ronneberg, 1995) I suggest three factors that should be investigated in future studies. These include (1) saliency of feedback, (2) familiarity between individuals in collaborative pairs, and (3) the size of the collaborative group. Each of these factors is discussed in turn.

F eedback

Varying the salience of feedback about performance could have a profound influence on collaborative search. In particular, varying feedback could influence the social dynamics of collaborative pairs. This prediction is based on the social loafing literature (see Karau \& Williams, 1993 for a review). Social loafing researchers have found that an individual $\llbracket$ s performance on simple tasks is poorer when others are working collectively (pooling their outputs) with that individual. This decrease in performance is often attributed to a reduction in motivation and effort when individuals work collectively compared to when they work as individuals. One factor that is thought to contribute to social loafing is a decrease in personal accountability; because it is difficult to identify individual contributions, people feel they can exert less effort without being noticed (e.g., Williams et al., 1981). Critically, personal accountability should depend on how salient the feedback is during task performance and how easily the feedback can be attributed to specific individuals in the group.

Varying the salience of the feedback in the collaborative condition could influence both response bias as well as sensitivity to the targets. Recall that in the collaborative condition the two individuals shared a response button, making the responses from each individual unidentifiable at the level of data analysis. However, feedback was provided after each trial making it obvious on target absent trials when an individual committed a false alarm. This might have increased feelings of personal accountability causing individuals to feel more personally responsible for the group outcome, and thus more reluctant to 
respond. This would have ultimately resulted in a conservative response bias in the collaborative condition. Pursing this line of reasoning further, if performance feedback was removed, individuals might feel less accountable causing them to respond more liberally. Alternatively, if the feedback was more explicit about which member of the pair made a mistake, this would increase feelings of personal responsibility, making partners even less likely to respond (i.e., become even more conservative). Thus, varying the salience of the feedback could radically influence the response bias. Of course, varying the saliency of feedback might affect an individual $\llbracket$ s ability to detect the target (i.e., sensitivity). It is possible that without feedback, an individual will sit back and rely on their partner】s performance. However, when feedback is provided that identifies which member of the pair was correct or incorrect, that same individual might pay closer attention, thus increasing their ability to detect the target.

Another social factor that might be affected by manipulating feedback is evaluation apprehension (e.g. Collaros \& Anderson, 1969). When working in groups, people worry about being negatively evaluated by the group if they make an error. To avoid embarrassment, they set a very high criterion for the quality of the contributions they choose to make. Evaluation apprehension might also help explain the conservative response bias obtained in the collaborative condition. If feedback was removed, evaluation apprehension will be greatly decreased, and individuals might be more likely to respond indicating that they saw the target. In contrast, if the salience of feedback was increased, individuals might be less likely to respond.

In addition to the social factors described above, feedback can also affect the cognitive aspects of collaborative search. Feedback allows the members of the pair to evaluate how well their task sharing strategies are working. In future studies I plan to vary the saliency of feedback to see how this affects a collaborative pair $\llbracket s$ ability to make use of effective task sharing strategies. 
Familiarity

Manipulating the level of familiarity the two members of the pair have with each other might also have an effect on the social and cognitive factors of collaborative search. From a social perspective, pairs of individuals that know each other (e.g., friends or spouses) might be less affected by evaluation apprehension. As such, collaborative pairs that are familiar with each other may be more likely to respond to targets than relatively unfamiliar collaborative pairs.

Level of familiarity might also have an impact on the cognitive aspects of task sharing. Pairs that are very familiar with each other know each other】s strengths and weaknesses and thus may be more effective at task sharing than are less familiar pairs. This type of advantage for familiar pairs is akin to the advantage familiar pairs have in memory tasks because they have information about how the other person $\llbracket$ s memory is represented or what they know (i.e., transactive memory; Wegner, Raymond, \& Erber, 1991). Interestingly, contrary to the present collaborative search results, many studies of collaborative recall find that collaborative groups perform poorer than nominal groups (i.e., collaborative inhibition; e.g. Weldon \& Bellinger, 1997). One proposed explanation for this collaborative inhibition is retrieval interference (e.g., Basden et al., 1997, Finlay, Hitch \& Meudell, 2000). Importantly, collaborative inhibition is decreased on memory tasks when friends or spouses are compared to strangers (Andersson \& Ronneberg, 1995). This might be due to the transactive memory that friends and spouses possess. This can lead to the development of a distributed memory system, such that they are able to divide responsibility for the encoding, storage, and retrieval of information from different areas according to their implicitly shared knowledge of each other. In memory tasks, this knowledge can be used to provide cues to help the other person retrieve an item from memory and also to know what your partner is more likely to remember (in turn reducing retrieval interference). Similarly, these processes can be used in the context of collaborative visual search to more effectively task share. Future research will compare 
individuals who know each other to randomly paired individuals to examine whether the more familiar pairs will exhibit more effective task sharing and increased ability to detect the targets.

Group Size

Group size is another factor that could have a large impact on collaborative search performance. According to social loafing research, increasing group size diffuses responsibility and decreases personal accountability resulting in group output below what would be expected based on the number of members in the group (e.g., Ingham et al., 1974; Petty, Harkins, Williams, \& Latane, 1977). Additionally, in the collaborative memory literature, as group size increases, more collaborative inhibition is seen. For example, when pairs of individuals perform memory recall tasks, collaboration either produces no benefit in recall tasks (Meudell, Hitch, \& Kirby, 1992; Meudell, Hitch, \& Boyle, 1995) or impairs recall relative to nominal pairs (Andersson \& Ronnberg, 1995, 1996). In groups of three or four, the collaborative groups consistently recall less than the nominal groups (eg. Weldon \& Bellinger, 1997, Basden et al., 1997).

A next step for collaborative search is to increase the group size to see how group performance is affected. According to social loafing research, personal accountability will decrease as group size increases, thus reducing the collaborative output. However, when there are more members in the group, there are more ways to divide the task (i.e., more task sharing). Due to these competing factors, it is unclear how search will be affected, and thus it would be interesting to manipulate group size in future research to see how collaborative performance is influenced by more group members.

\subsubsection{Real W orld Implications}

Finally, it is worth noting that the present findings also have important implications for search in real world situations. For instance, consider security searches at airports. Because such searches are typically conducted by multiple individuals, an obvious question is whether they should be conducted by individuals working together (collaborative pairs) or working apart (nominal pairs)? The present findings 
suggest that the answer depends on whether it is desirable to detect as many targets as possible or whether it is more important to minimize false alarms. If it is preferable to detect as many targets as possible, search should be conducted by individuals working independently because nominal pairs have a greater hit rate than do collaborative pairs. If, on the other hand, it is more important to minimize false alarms, security search should be conducted by collaborative pairs. Thus, the present findings have direct bearing on the sorts of policies and procedures that might be implemented in real world contexts. More generally, I believe that including collaboration in studies of visual search brings research one step closer to the real world. 


\section{R eferences}

Andersson, J., \& Ronnberg, J. (1995). Recall suffers from collaboration: Joint recall effects of friendship and task complexity. Applied Cognitive Psychology, 9(3), 199-211.

Andersson, J., \& Ronnberg, J. (1996). Collaboration and memory: Effects of dyadic retrieval on different memory tasks. Applied Cognitive Psychology, 10(2), 171-181.

Basden, B.H., Basden, D.R., Bryner, S., \& Thomas, R.L. (1997). A comparison of group and individual remembering: Does collaboration disrupt retrieval strategies? J ournal of Experimental Psychology: Learning, M emory, and Cognition, 23(5), 1176-1189.

Bundesen, C. (1990). A theory of visual attention. Psychological Review, 97(4), 523-547.

Clark, A. 1999. An embodied cognitive science? Trends in Cognitive Science, 9, 345-351.

Collaros, P.A., \& Anderson, L.R. (1969). Effect of perceived expertise upon creativity of members of brainstorming groups. J ournal of Applied Psychology, 53(2), 159-163.

Donaldson, W. (1992). Measuring recognition memory. J ournal of Experimental P sychology: General, 121(3), 275-277.

Duncan, J., \& Humphreys, G.W. (1989). Visual search and stimulus similarity. Psychological Review, 96(3), 433-458.

Findlay, J.M., \& Gilchrist, I.D. (1998). Eye guidance and visual search. In G. Underwood (Ed.), Eye guidance in reading and scene perception (pp. 295-312). Amsterdam: Elsevier Science.

Finlay, F., Hitch, G.J., \& Meudell, P.R. (2000). Mutual inhibition in collaborative recall: Evidence for a retrieval-based account. J ournal of Experimental Psychology: Learning, M emory, and Cognition, $26,1556-1567$.

Harkins, S.G., \& Jackson, J.M. (1985). The role of evaluation in eliminating social loafing. Personality and Social Psychology Bulletin, 11, 575-584. 
Harkins, S.G., \& Petty, R.E. (1982). Effects of task difficulty and task uniqueness on social loafing. J ournal of Personality and Social Psychology, 43, 1214-1229.

Harkins, S.G., \& Szymanski, K. (1989). Social loafing and group evaluation. J ournal of Personality and Social Psychology, 10, 934-941.

Hooge, I.T. \& Erkelens, C.J. (1996). Control of fixation duration in a simple search task. Perception \& Psychophysics, 58(7), 969-976.

Horowitz, T.S., \& Wolfe, J.M. (1998). Visual search has no memory. Nature, 394, 575-577.

Humphreys, G., \& Muller, H. (1993). SEarch via recursive rejection (SERR): A connectionist model of visual search. Cognitive Psychology, 25(1), 43-110.

Hutchins, E. (1995). Cognition in the Wild. Cambridge, MA: MIT Press.

Ingham, A.G., Levinger, G., Graves, J., \& Peckman, V. (1974). The Ringlemann effect: Studies of group size and group performance. J ournal of Personality and Social Psychology, 10, 371-384.

Jack, A.I., \& Roepstorff, A. (2002). Introspection and cognitive brain mapping: from stimulus-response to script-report. Trends in Cognitive Sciences, 6, 333-339.

Karau, S.J., \& Williams, K.D. (1993). Social loafing: A meta-analytic review and theoretical integration. J ournal of Personality and Social Psychology, 65(4), 681-706.

Kingstone, A., Smilek, D., Ristic, J., Kellend Friesen, C., \& Eastwood, J.D. (2003). Attention, researchers! It is time to take a look at the real world. Current D irections in P sychological Science, 12(5), 176-180.

Klein, R., \& Farrell, M. (1989). Search performance without eye movements. Perception \& Psychophysics, 46(5), 476-482.

Luck, S.J., \& Hillyard, S.A. (1994). Electrophysiological correlates of feature analysis during visual search. P sychophysiology, 31(3), 291-308. 
Meudell, P.R., Hitch, G.J., \& Boyle, M.M. (1995). Collaboration in recall: Do pairs of people cross-cue each other to produce new memories? Quarterly J ournal of Experimental P sychology, 48A(1), 141152.

Meudell, P.R., Hitch, G.J. \& Kirby, P. (1992). Are two heads better than one? Experimental investigations of the social facilitation of memory. Applied Cognitive Psychology, 6, 525-543.

Petty, R.E., Harkins, S.G., Williams, K.D., \& Latane, B. (1977). The effects of group size on cognitive effort and evaluation. Personality and Social P sychology Bulletin, 3(4), 579-582.

Pickering, M.J., \& Garrod, S. (2004). Toward a mechanistic psychology of dialogue. Behavioral and Brain Sciences, 27(2), 169-226.

Roepstorff, A., \& Frith, C. (2004). What s at the top in the top-down control of action? Script-sharing and „top-top $\square$ control of action in cognitive experiments. P sychological Research, 68, 189-198.

Rolls, E.T., \& Deco, G. (2002). Computational neuroscience of vision. New York, NY: Oxford University Press.

Ross, M., Spencer, S.J., Linardatos, L., Lam, K.C., \& Perunovic, M. (2004). Going shopping and identifying landmarks: Does collaboration improve older people's memory? Applied Cognitive P sychology, 18, 683-696.

Sebanz, N., Bekkering, H., \& Knoblich, G. (2006). Joint action: bodies and minds moving together. Trends in Cognitive Sciences, 10(2), 70-76.

Thelen, E., \& Smith, L.B. (1994). A dynamic systems approach to the development of cognition and action. Cambridge, MA: Bradford Books/MIT Press.

Tomasello, M. (1995). Joint attention as social cognition. In C. Moore and P. Dunham (Eds.), J oint attention: Its origins and role in development (pp. 103-130). Hillsdale, NJ: Erlbaum.

Treisman, A., \& Gelade, G. (1980). A feature integration theory of attention. Cognitive Psychology, 12, 97-136. 
Wegner, D.M., Raymond, P., \& Erber, R. (1991). Transactive memory in close relationships. J ournal of Personality and Social P sychology, 61, 923-929.

Weldon, M.S., \& Bellinger, K.D. (1997). Collective memory: Collaborative and individual processes in remembering. J ournal of Experimental Psychology: Learning, M emory, and Cognition, 23(5), $1160-1175$.

Williams, K., Harkins, S.G., \& Latane, B. (1981). Identifiability as a deterrent to social loafing: Two cheering experiments. J ournal of Personality and Social Psychology, 40(2), 303-311.

Wolfe, J.M. (1994). Guided search 2.0: A revised model of visual search. Psychonomic Bulletin \& Review, 1(2), 202-238.

Wolfe, J.M., Friedman-Hill, S.R., Stewart, M.I. \& O\Connell, K.M. (1992). The role of categorization in visual search for orientation. J ournal of Experimental Psychology: Human Perception and Performance, 18(1), 34-49.

Wolfe, J.M., \& Horowitz, T.S. (2004). What attributes guide the deployment of visual attention and how do they do it? Nature Reviews Neuroscience, 5(6), 495-501.

Zajonc, R.B. (1965). Social facilitation. Science, 149, 269-274.

Zelinsky, G.J., \& Sheinberg, D.L. (1997). Eye movements during parallel-serial visual search. J ournal of Experimental Psychology: Human Perception and Performance, 23(1), 244-262. 


\section{Note}

1. I varied exposure duration between-pairs and set size with-in participants in order to ensure that I had a wide range of hits and farms alarms. I decided not to titrate exposure duration for each individual because this would make the experiment different for each member of the nominal pair, making it impossible to

pool their responses. In both the nominal and collaborative conditions, search performance was averaged across exposure duration and set size and no formal analyses of these factors were conducted. 
Appendix A:

Mean Proportion of Hits, Proportion of False Alarms, Sensitivity and R esponse Bias in the Collaborative and Nominal Conditions for E ach Pair in Experiment 1

\begin{tabular}{|c|c|c|c|c|c|c|c|c|}
\hline \multicolumn{5}{|c|}{ Collaborative } & \multicolumn{4}{|c|}{ Nominal } \\
\hline \multirow[b]{2}{*}{ Pair \# } & \multicolumn{3}{|c|}{ False } & \multirow{2}{*}{$\begin{array}{c}\text { Response } \\
\text { Bias }\end{array}$} & \multicolumn{3}{|c|}{ False } & \multirow{2}{*}{$\begin{array}{c}\text { Response } \\
\text { Bias }\end{array}$} \\
\hline & Hits & Alarms & Sensitivity & & Hits & Alarms & Sensitivity & \\
\hline 1 & .767 & .017 & .935 & .894 & .933 & .417 & .860 & -.818 \\
\hline 2 & .817 & .183 & .888 & .000 & .883 & .433 & .826 & -.705 \\
\hline 3 & .950 & .050 & .974 & .000 & .883 & .300 & .873 & -.529 \\
\hline 4 & .850 & .017 & .957 & .825 & .983 & .083 & .974 & -.686 \\
\hline 5 & .833 & .050 & .941 & .583 & .983 & .300 & .918 & -.924 \\
\hline 6 & .617 & .200 & .799 & .426 & .900 & .767 & .680 & -.935 \\
\hline 7 & .433 & .200 & .708 & .679 & .850 & .733 & .644 & -.879 \\
\hline 8 & .683 & .133 & .860 & .502 & .950 & .417 & .869 & -.863 \\
\hline 9 & .817 & .550 & .730 & -.690 & .967 & .800 & .751 & -.983 \\
\hline 10 & .750 & .233 & .841 & .045 & .900 & .533 & .798 & -.823 \\
\hline 11 & .850 & .200 & .894 & -.172 & .933 & .733 & .741 & -.949 \\
\hline 12 & .933 & .067 & .964 & .000 & .983 & .350 & .905 & -.939 \\
\hline 13 & .917 & .283 & .894 & -.626 & .967 & .533 & .844 & -.941 \\
\hline 14 & .817 & .250 & .862 & -.195 & 1.000 & .400 & .900 & -1.000 \\
\hline 15 & .817 & .350 & .822 & -.412 & .933 & .917 & .554 & -.987 \\
\hline 16 & .850 & .283 & .864 & -.383 & 1.000 & .500 & .875 & -1.000 \\
\hline 17 & .800 & .067 & .926 & .556 & .933 & .483 & .838 & -.858 \\
\hline 18 & .933 & .133 & .945 & -.366 & .967 & .200 & .938 & -.758 \\
\hline 19 & .833 & .317 & .844 & -.397 & .917 & .733 & .722 & -.936 \\
\hline 20 & .900 & .067 & .955 & .217 & .950 & .200 & .932 & -.652 \\
\hline 21 & .850 & .067 & .940 & .424 & .852 & .533 & .765 & -.737 \\
\hline 22 & .733 & .000 & .933 & 1.000 & .900 & .500 & .811 & -.800 \\
\hline 23 & .783 & .000 & .946 & 1.000 & .917 & .717 & .731 & -.931 \\
\hline 24 & .817 & .150 & .900 & .120 & .967 & .400 & .883 & -.902 \\
\hline M ean & .806 & .161 & .888 & .168 & .936 & .499 & .818 & -.856 \\
\hline
\end{tabular}


Appendix B:

Mean Proportion of Hits, Proportion of False Alarms, Sensitivity and R esponse Bias in the Collaborative and Nominal Conditions for E ach Pair in Experiment 2

\begin{tabular}{|c|c|c|c|c|c|c|c|c|}
\hline \multirow[b]{3}{*}{ Pair \# } & \multicolumn{4}{|c|}{ Collaborative } & \multicolumn{4}{|c|}{ Nominal } \\
\hline & & False & & Response & & False & & Response \\
\hline & Hits & Alarms & Sensitivity & Bias & Hits & Alarms & Sensitivity & Bias \\
\hline 1 & .683 & .400 & .722 & -.180 & .917 & .600 & .784 & -.886 \\
\hline 2 & .600 & .133 & .829 & .625 & .817 & .517 & .747 & -.653 \\
\hline 3 & .717 & .200 & .842 & .225 & .750 & .600 & .644 & -.636 \\
\hline 4 & .600 & .233 & .772 & .373 & .767 & .417 & .764 & -.402 \\
\hline 5 & .533 & .283 & .704 & .378 & .850 & .783 & .597 & -.907 \\
\hline 6 & .433 & .067 & .810 & .896 & .750 & .617 & .631 & -.657 \\
\hline 7 & .833 & .233 & .876 & -.207 & .800 & .500 & .744 & -.600 \\
\hline 8 & .600 & .067 & .865 & .806 & .933 & .517 & .827 & -.875 \\
\hline 9 & .700 & .067 & .896 & .714 & .900 & .833 & .619 & -.957 \\
\hline 10 & .700 & .067 & .896 & .714 & .750 & .150 & .876 & .308 \\
\hline 11 & .767 & .050 & .922 & .705 & .950 & .650 & .793 & -.945 \\
\hline 12 & .350 & .250 & .605 & .696 & .650 & .367 & .721 & -.036 \\
\hline 13 & .783 & .050 & .927 & .680 & .867 & .450 & .810 & -.683 \\
\hline 14 & .483 & .117 & .793 & .780 & .783 & .217 & .862 & .000 \\
\hline 15 & .850 & .183 & .900 & -.120 & .833 & .367 & .824 & -.486 \\
\hline 16 & .683 & .050 & .898 & .796 & .933 & .183 & .930 & -.517 \\
\hline 17 & .717 & .017 & .922 & .918 & .717 & .133 & .872 & .440 \\
\hline 18 & .683 & .083 & .883 & .672 & .867 & .483 & .796 & -.718 \\
\hline 19 & .567 & .050 & .864 & .871 & .483 & .600 & .367 & -.168 \\
\hline 20 & .750 & .567 & .667 & -.594 & .950 & .850 & 693 & -.982 \\
\hline 21 & .783 & .067 & .921 & .590 & .817 & .583 & .711 & -.724 \\
\hline 22 & .700 & .133 & .866 & .472 & .883 & .450 & .820 & -.722 \\
\hline 23 & .867 & .017 & .961 & .802 & .933 & .383 & .870 & -.794 \\
\hline 24 & .750 & .050 & .918 & .727 & .733 & .100 & .892 & .532 \\
\hline Mean & .672 & .143 & .844 & .514 & .818 & .473 & .758 & -.503 \\
\hline
\end{tabular}


Appendix C:

Mean Proportion of Hits, Proportion of False Alarms, Sensitivity and Response Bias in the Collaborative and Nominal Conditions for E ach Pair in Experiment 3

\begin{tabular}{|c|c|c|c|c|c|c|c|c|}
\hline & \multicolumn{4}{|c|}{ Collaborative } & \multicolumn{4}{|c|}{ Nominal } \\
\hline \multirow[b]{2}{*}{ Pair \# } & \multicolumn{3}{|c|}{ False } & \multirow{2}{*}{$\begin{array}{c}\text { Response } \\
\text { Bias }\end{array}$} & \multicolumn{3}{|c|}{ False } & \multirow{2}{*}{$\begin{array}{c}\text { Response } \\
\text { Bias }\end{array}$} \\
\hline & Hits & Alarms & Sensitivity & & Hits & Alarms & Sensitivity & \\
\hline 1 & .783 & .533 & .714 & -.610 & .950 & .800 & .727 & -.974 \\
\hline 2 & .750 & .200 & .855 & .143 & .967 & .400 & .883 & -.902 \\
\hline 3 & .717 & .067 & .901 & .694 & .950 & .500 & .843 & -.900 \\
\hline 4 & .850 & .217 & .888 & -.221 & .950 & .183 & .936 & -.620 \\
\hline 5 & .800 & .233 & .862 & -.098 & .883 & .833 & .589 & -.949 \\
\hline 6 & .883 & .000 & .971 & 1.000 & .967 & .067 & .974 & -.349 \\
\hline 7 & .750 & .183 & .862 & .195 & .917 & .617 & .777 & -.893 \\
\hline 8 & .833 & .150 & .906 & .062 & .933 & .367 & .875 & -.780 \\
\hline 9 & .967 & .217 & .933 & -.778 & 1.000 & .450 & .888 & -1.000 \\
\hline 10 & .750 & .183 & .862 & .195 & .917 & .333 & .878 & -.692 \\
\hline 11 & .883 & .033 & .960 & .586 & .850 & .213 & .890 & -.211 \\
\hline 12 & .833 & .217 & .882 & -.161 & .883 & .250 & .890 & -.432 \\
\hline 13 & .900 & .100 & .944 & .000 & .950 & .417 & .869 & -.863 \\
\hline 14 & 1.000 & .133 & .967 & -1.000 & .967 & .150 & .951 & -.673 \\
\hline 15 & .800 & .033 & .938 & .758 & .917 & .283 & .894 & -.626 \\
\hline 16 & .983 & .083 & .974 & -.686 & .917 & .233 & .909 & -.540 \\
\hline 17 & .767 & .350 & .796 & -.278 & .833 & .517 & .759 & -.685 \\
\hline 18 & .367 & .017 & .828 & .981 & .833 & .467 & .782 & -.628 \\
\hline 19 & .883 & .333 & .862 & -.582 & .883 & .633 & .741 & -.858 \\
\hline 20 & .650 & .233 & .796 & .278 & .900 & .583 & .778 & -.853 \\
\hline 21 & .833 & .367 & .824 & -.486 & .933 & .783 & .713 & -.961 \\
\hline 22 & .783 & .117 & .901 & .354 & .850 & .550 & .755 & -.748 \\
\hline 23 & .817 & .217 & .875 & -.104 & .917 & .567 & .797 & -.870 \\
\hline 24 & .767 & .067 & .916 & .620 & .850 & .183 & .900 & -.120 \\
\hline M ean & .806 & .178 & .884 & .036 & .913 & .432 & .833 & -.714 \\
\hline
\end{tabular}

